\title{
Minimally cultured bone marrow mesenchymal stem cells ameliorate fibrotic lung injury
}

\author{
M. Kumamoto*,\#, , T. Nishiwaki*,
}

ABSTRACT: Clinical use of bone marrow mesenchymal stem cells (BMMSCs) holds great promise for regenerative medicine in intractable lung diseases, such as lung fibrosis or acute respiratory distress syndrome. However, a severe obstacle to the clinical application of BMMSC transplantation is the time-consuming, laborious processes required for cell culture. In order to evaluate the clinical applicability of BMMSC transplantation, we tested whether engraftment of minimally cultured BMMSCs ameliorates progressive fibrotic lung injury.

Differences between murine BMMSCs cultured for $2 \mathrm{~h}$ (2-h adherent BMMSCs) and conventionally (9-day) cultured BMMSCs were examined in vitro. The effects of grafting either type of BMMSCs on fibrotic lung injury were then assessed by transfer experiments in a murine bleomycin-induced lung fibrosis model, in which donor cells were administered 3 days after challenge.

2-h adherent BMMSCs were smaller, less granular, possessed higher proliferative capacity and expressed higher levels of several stem cell markers and chemokine receptors than 9-day cultured BMMSCs, but lower type I procollagen, $\alpha$-smooth muscle actin, tumour necrosis factor- $\beta$ and oncogenic transcription factor c-Myc, suggesting that they may be advantageous for cellbased therapy compared with 9-day cultured BMMSCs. Grafting 2-h adherent BMMSCs ameliorated inflammatory and fibrotic lung disorders, and reduced mortality equally well or better than 9-day cultured BMMSCs.

Minimally cultured BMMSCs can substitute for conventionally cultured BMMSCs and will be a promising cell source for the treatment of acute fibrotic lung injury.

KEYWORDS: Acute lung injury, bone marrow mesenchymal stem cells, cell culture process, lung fibrosis

ince their first description in 1976 by FRIEDENSTEIN et al. [1], bone marrow mesenchymal stem cells (BMMSCs) have received much attention as a possible cell source for regenerative medicine. Although BMMSCs had primarily been considered as a progenitor population only for mesodermal cell lines, recent studies have revealed their ability to differentiate into ectodermal and endodermal cell lineages. Accordingly, increasing interest has been focused on the use of these cells for diverse clinical applications for repairing damaged organs.

In some regards, such as bioethical issues or problems with immunological rejection, autologous bone marrow transplantation is likely to be less problematic than allogeneic bone marrow transplantation for clinical application of stem cell-based therapy. However, the requirement for cell culture processes limits its general applicability for acutely fatal diseases because of the necessity for emergency intervention. In addition, previous studies have highlighted other issues regarding the cultivation of BMMSCs, including laboratory contamination, influence on proliferating, differentiating or homing potential [2-5], and acquisition of tumorigenic properties [6, 7].

In the case of the lung, several animal models have suggested that bone marrow-derived cells have therapeutic potential for intractable diseases, including lung fibrosis [8,9], emphysema [10] and acute lung injury (ALI) [11]. Although the effectiveness of BMMSC treatment for

\section{AFFILIATIONS}

*Dept of Molecular Preventive Medicine, School of Medicine, University of Tokyo, Tokyo,

\#Second Dept of Internal Medicine, Nara Medical University, Nara, Japan. "These investigators contributed equally to this article.

CORRESPONDENCE

K. Matsushima

Dept of Molecular Preventive Medicine, School of Medicine University of Tokyo

7-3-1 Hongo

Bunkyo-ku

Tokyo 113-0033

Japan

E-mail: koujim@m.u-tokyo.ac.jp

Received:

Aug 202008

Accepted after revision:

March 062009

First published online:

March 262009 
bleomycin-induced lung injury, an animal model representing lethal fibrotic lung injury such as acute respiratory distress syndrome (ARDS), has been demonstrated in two reports, both of which used cultured BMMSCs to evaluate the repair capacity of damaged lung $[8,9]$. Therefore, in order to extend the potential for clinical application of BMMSC-based treatment for ALI, we investigated whether the culture duration of BMMSCs could be reduced to a minimum, while maintaining the healing potential.

In the present study, the donor bone marrow cells were cultured for only $2 \mathrm{~h}$; these cells are termed 2-h adherent BMMSCs. They were compared with conventionally (9-day) cultured BMMSCs with regard to their cellular and biological characteristics, and their restorative capacity for acute fibrotic lung injury under strict conditions, namely cell administration on day 3 of bleomycin challenge, mimicking an actual clinical setting.

We have not only verified the clinical feasibility of 2-h adherent BMMSC-based treatment for fibrotic lung injury, but also provide evidence for the comparable grafting potential of 2-h adherent BMMSCs and 9-day cultured BMMSCs.

\section{MATERIALS AND METHODS \\ Mice}

Female C57BL/6J mice (6-8 weeks old) were purchased from Japan SLC (Shizuoka, Japan) or CLEA Japan (Tokyo, Japan), and kept under specific pathogen-free conditions in the animal care facility of the Department of Molecular Preventive Medicine, School of Medicine, University of Tokyo, Tokyo, Japan. All experiments complied with approved animal care protocols of the University of Tokyo.

\section{Isolation of murine 2-h adherent BMMSCs and 9-day cultured BMMSCs}

To isolate 2-h adherent BMMSCs, mouse bone marrow cells were plated at $1 \times 10^{6} \mathrm{cells} \cdot \mathrm{cm}^{-2}$ in Dulbecco's modified Eagle's medium (DMEM) containing 2\% fetal bovine serum (FBS) (Invitrogen, Carlsbad, CA, USA) and cultured at $37^{\circ} \mathrm{C}$ in $5 \%$ carbon dioxide. After $2 \mathrm{~h}$, adherent cells were harvested by gentle scraping and incubated with biotinylated anti-CD11b, anti-CD45 and anti-CD31 antibodies (BD Pharmingen, San Diego, CA USA), followed by purification of cells negative for CD11b, CD45 and CD31 (CD11b-CD45-CD31- cells) using a magnet-activated cell sorting system (Miltenyi Biotech, Gladbach, Germany). 9-day cultured BMMSCs were isolated as described previously [9].

For morphological analysis, the cells were stained with DiffQuik (Baxter, Miami, FL, USA).

\section{Differentiation of 2-h adherent BMMSCs}

For osteogenesis, 2-h adherent cells were incubated in DMEM supplemented with $10 \%$ FBS, penicillin/streptomycin, $10 \mathrm{mM}$ glycerophosphate (Calbiochem, San Diego, CA, USA), $10^{-8} \mathrm{M}$ dexamethasone (Sigma, St Louis, MO, USA) and $0.5 \mu \mathrm{M}$ ascorbic acid (Sigma) for 10 days. The cells were fixed with $10 \%$ formalin for $10 \mathrm{~min}$ at room temperature and stained with 1\% Alizarin Red S (Sigma) for $5 \mathrm{~min}$ at room temperature.

For adipogenesis, 2-h adherent cells were incubated in DMEM supplemented with $10 \%$ FBS, penicillin/streptomycin, $5 \mu \mathrm{g} \cdot \mathrm{mL}^{-1}$ insulin (Sigma) and $10^{-6} \mathrm{M}$ dexamethasone (Sigma) for 10 days. The cells were fixed with $10 \%$ formalin for $10 \mathrm{~min}$ at room temperature and washed in $60 \%$ isopropanol, and subsequently incubated for $10 \mathrm{~min}$ with Oil-Red O to visualise lipid droplets. Cells were then washed in isopropanol.

Control cells were incubated in DMEM supplemented with $10 \%$ FBS and penicillin/streptomycin. After 10 days, cells were fixed and stained with Alizarin Red S or Oil-Red O.

\section{Cell cycle analysis}

Cell cycle analysis was performed using bromodeoxyuridine (BrdU) flow kits (BD Pharmingen), according to the manufacturer's instructions. Briefly, BrdU was added to cell cultures at a final concentration of $10 \mu \mathrm{M} 45 \mathrm{~min}$ prior to harvesting. BrdUpulsed cells were stained for surface markers, and then fixed and permeabilised by BD Cytofix/Cytoperm Buffer. To expose incorporated BrdU, cells were incubated with DNase for $1 \mathrm{~h}$ at $37^{\circ} \mathrm{C}$, and then stained with fluorescein isothiocyanate-conjugated anti-BrdU monoclonal antibody. After total DNA was stained by incubation with 7-aminoactinomycin (7-AAD) solution, cells were analysed by flow cytometry.

\section{Total RNA isolation and real-time RT-PCR}

Total RNA was isolated from 2-h adherent BMMSCs, 9-day cultured BMMSCs and lung specimens using RNAzol B (TelTest, Friendswood, TX, USA), according to the manufacturer's instructions. It was then reverse transcribed into cDNA and the expression of mRNA was determined by real-time quantitative PCR using the Power SYBR Green PCR Master Mix (Applied Biosystems, Foster City, CA, USA) and the 7500 Real Time PCR System (Applied Biosystems). Primer sequences are given in the online supplementary data. Threshold cycle for glyceraldehyde 3-phosphate dehydrogenase (GAPDH) was used to normalise the samples.

\section{Bleomycin-induced lung injury followed by administration of BMMSCs}

Mice were intratracheally injected with $3 \mathrm{mg} \cdot \mathrm{kg}^{-1}$ bleomycin sulphate (Nipponkayaku, Tokyo, Japan) in PBS under light anaesthesia. $5 \times 10^{5}$ BMMSCs were administrated into the tail vein 3 days later. Animals were killed 10 days after bleomycin exposure, and then bronchoalveolar lavage (BAL) was collected. After perfusion with PBS, left lungs were removed and used for RNA isolation and right lungs were used for morphometric analysis.

To evaluate the effect of BMMSCs on survival in bleomycintreated mice, animals were given $4 \mathrm{mg} \cdot \mathrm{kg}^{-1}$ bleomycin, because treatment with PBS after $3 \mathrm{mg} \cdot \mathrm{kg}^{-1}$ bleomycin injection resulted in a high survival rate.

\section{Collagen assay}

Lung collagen content was determined by assaying total soluble collagen using the Sircol Collagen Assay kit (Biocolor, Newton Abbey, UK), according to the manufacturer's instructions. Briefly, the desiccated whole left lungs were homogenised in $0.5 \mathrm{M}$ acetic acid containing $1 \mathrm{mg}$ pepsin/10 mg tissue residue. Each sample was incubated for $24 \mathrm{~h}$ at $4{ }^{\circ} \mathrm{C}$ with stirring. After centrifugation, each supernatant was assayed. Sircol dye reagent that binds to collagen was added to each sample and then mixed for $30 \mathrm{~min}$. After centrifugation, the pellet was suspended in the alkali reagent and read at $550 \mathrm{~nm}$ by a spectrophotometer. Collagen standard 
solutions were utilised to construct a standard curve. Collagen contents obtained with this method correlate well with the hydroxyproline content, according to the manufacturer's data.

\section{Statistical analysis}

All data are expressed as mean $\pm \mathrm{SD}$. Comparisons between the two groups were analysed using paired t-tests. Survival curves were derived by the Kaplan-Meier method and compared via generalised Wilcoxon test. A p-value $<0.05$ was considered statistically significant.

\section{RESULTS \\ Isolation of murine 2-h adherent BMMSCs and 9-day cultured BMMSCs}

The plastic-adherent cells cultured for 9 days consisted of three morphologically distinct cell types, including large polygonal fibroblast-like cells, which were previously suggested to be mesenchymal stem cells (fig. 1a) $[12,13]$. In order to determine whether the cells with mesenchymal stem cell properties were involved at an early stage of cell culture, we started the differentiative induction of bone marrow adherent cells $2 \mathrm{~h}$ after the plating. For the first 10 days, only large polygonal fibroblastlike cells showed intracellular calcium-rich mineralised deposition or lipid droplets, suggesting the existence of BMMSCs in the initial phase of cell culture (fig. $1 \mathrm{~b}$ and $\mathrm{d}$ ). Because the cells with myeloid/endothelial morphologies failed to differentiate into mesenchymal lineages, sorted 2-h adherent CD11b-CD45CD31- cells and their counterparts were further separately cultured. After 14 days of culture, the population derived from 2-h adherent CD11b-CD45-CD31- cells showed homogeneously widespread fibroblast-like morphology (fig. 1g), suggesting that these cells were progenitors of 9-day cultured BMMSCs. Conversely, the population of 2-h adherent cells positive for CD11b, CD45 or CD31 (CD11b+/CD45+/CD31+) showed two distinct cell morphologies, namely small rounded cells and small stellate cells after an additional 14 days of culture (fig. 1f).

These observations demonstrated that 2-h adherent CD11bCD45-CD31- bone marrow cells were multipotent mesenchymal stromal cells and the progenitors of established population of BMMSCs, and we accordingly regarded these cells as 2-h adherent BMMSCs. Further morphological analysis showed that 2-h adherent CD11b+/CD45+/CD31+ cells comprised a heterogeneous cell population containing mononuclear cells and polymorphonuclear cells (fig. $2 a$ and b), and these cells enlarged to approximately twice their original diameter after 9 days of culture (fig. 2c and d). Conversely, 2-h adherent BMMSCs were small rounded cells (fig. 2e and f), while 9-day cultured BMMSCs were large cells with abundant cytoplasm and nuclei in eccentric positions. (fig. $2 \mathrm{~g}$ and $\mathrm{h}$ ). 9-day cultured BMMSCs had more than four times the diameter of 2-h adherent BMMSCs.

Flow cytometric data also demonstrated that whole and CD11bC45-CD31- -gated bone marrow adherent cells increased progressively in size and granularity as a function of the duration of culture (fig. 1a and b in the online supplementary material).

\section{Comparison of murine 2-h adherent BMMSCs with 9-day cultured BMMSCs in vitro}

In order to assess the proliferative capacities of 2-h adherent BMMSCs and 9-day cultured BMMSCs, we analysed cell cycle

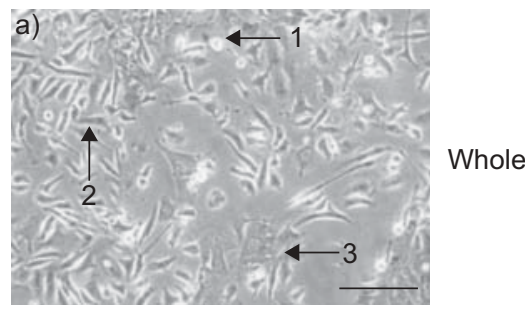

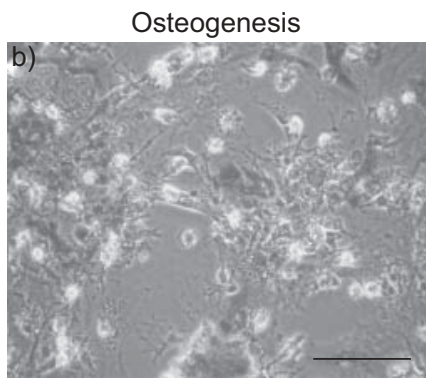

Adipogenesis

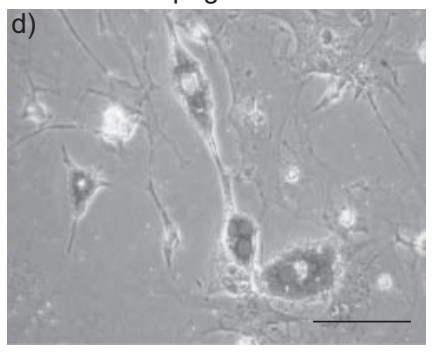

Positive
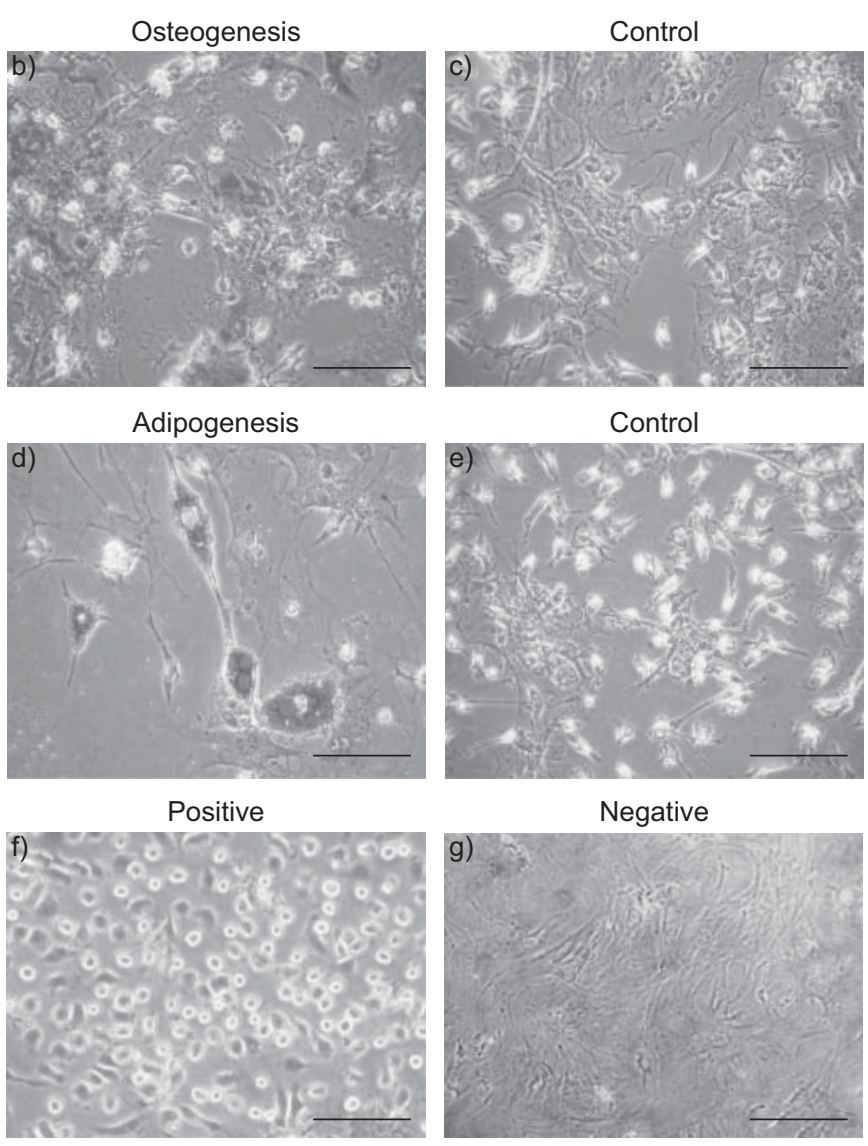

Control
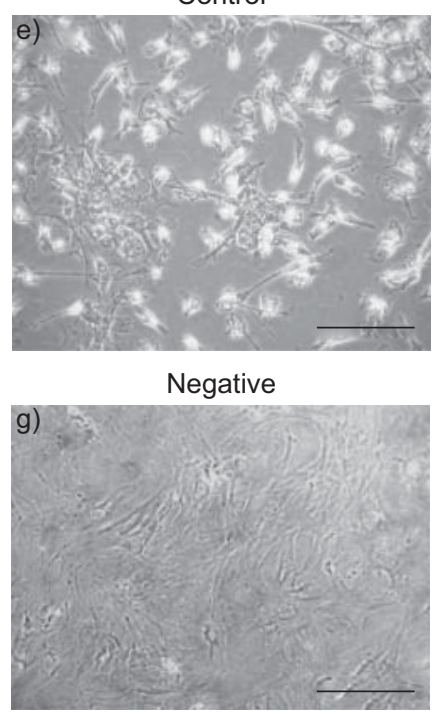

FIGURE 1. Whole bone marrow cell and 2-h adherent bone marrow mesenchymal stem cell (BMMSC) cultures, and the differentiation of 2-h adherent BMMSCs into mesenchymal lineage. 2-h adherent CD11b-CD45-CD31- cells (2-h adherent BMMSCs) showed homogeneously 9-day cultured BMMSC-like morphology after 14 more days of culture, and differentiated into adipogenic and osteogenic lineages when treated with adipogenic or osteogenic medium for 10 days. a) Whole bone marrow cells were cultured for 9 days. Plastic-adherent cells consisted of three morphologically distinct cell types: 1) small rounded cells; 2) small stellate cells; 3) large polygonal fibroblast-like cells. b) Calcium deposits were revealed by staining with Alizarin Red S. d) Accumulation of lipid droplets within the cells was revealed by staining with Oil Red O. c and e) Control cells did not reveal either calcium deposits or lipids. f) 2-h adherent CD11b+/CD31+/CD45+ cells were cultured for 14 days. The culture contained two distinct cell types: small rounded cells and small stellate cells. g) 2-h adherent CD11b-CD31-CD45- cells (2-h adherent BMMSCs) were cultured for additional 14 days. All cells showed homogenous fibroblastoid morphology. Scale bars $=200 \mu \mathrm{m}$.

phases using flow cytometry with a combination of BrdU and 7 -AAD. There was no dramatic reduction in the fraction of 2-h or 9-day adherent whole bone marrow cells in the $S$ phase ( $35.5 \%$ and $23.6 \%$, respectively; fig. $2 \mathrm{i}$ and $\mathrm{k}$ ). In contrast, gating on CD11b-CD45-CD31- cells demonstrated that the 
$2 \mathrm{~h}$

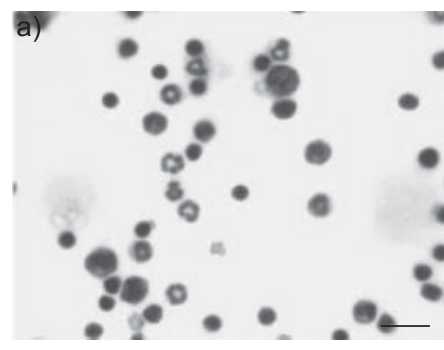

2-h adherent BMMSC

e)

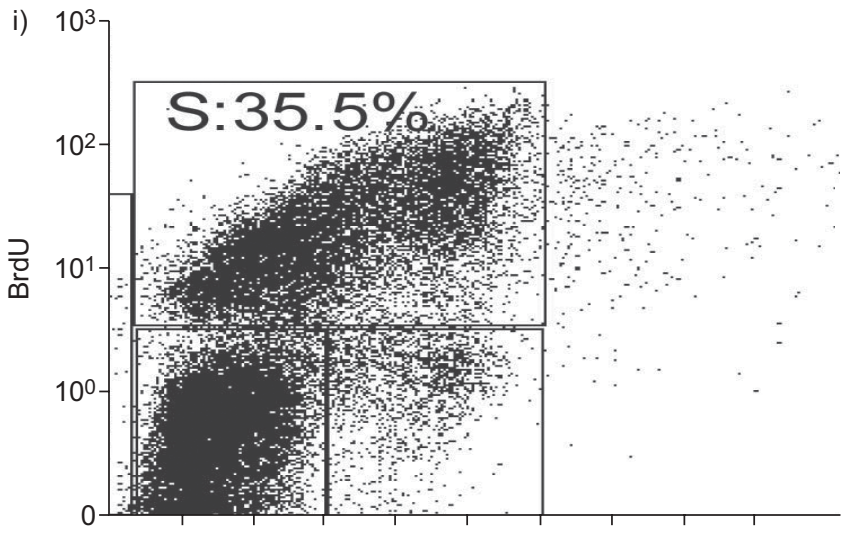

k) 1

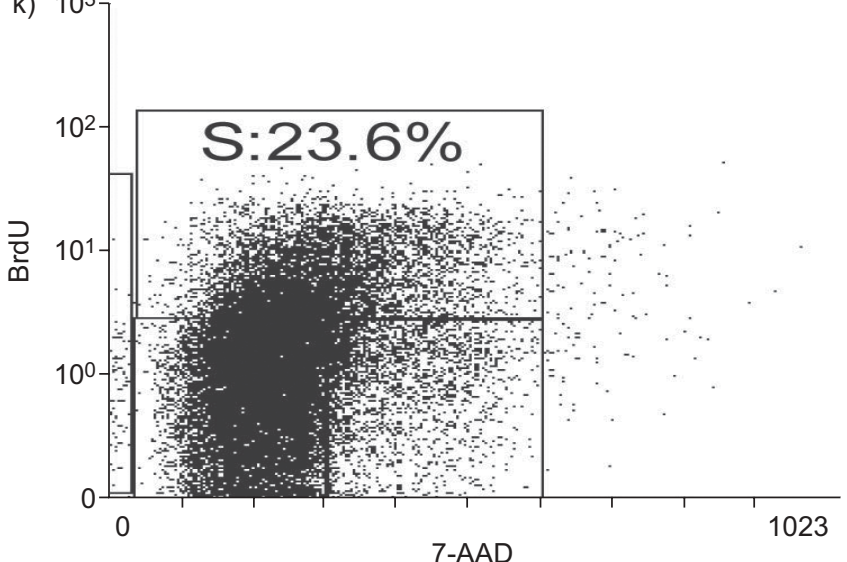

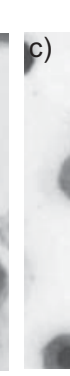
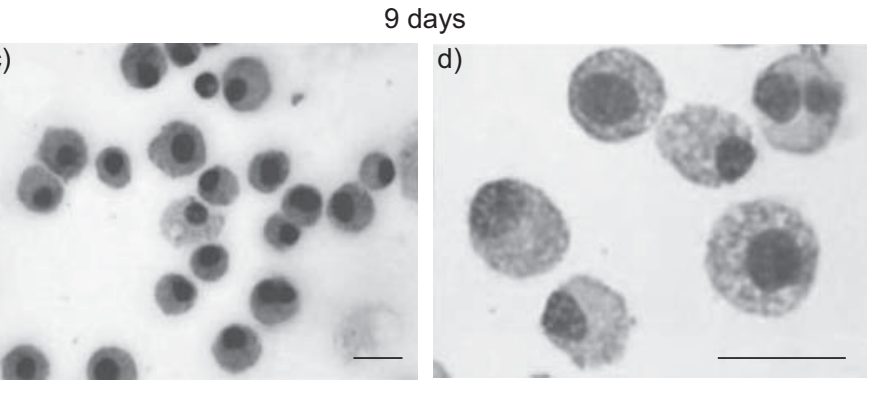

9-day cultured BMMSC
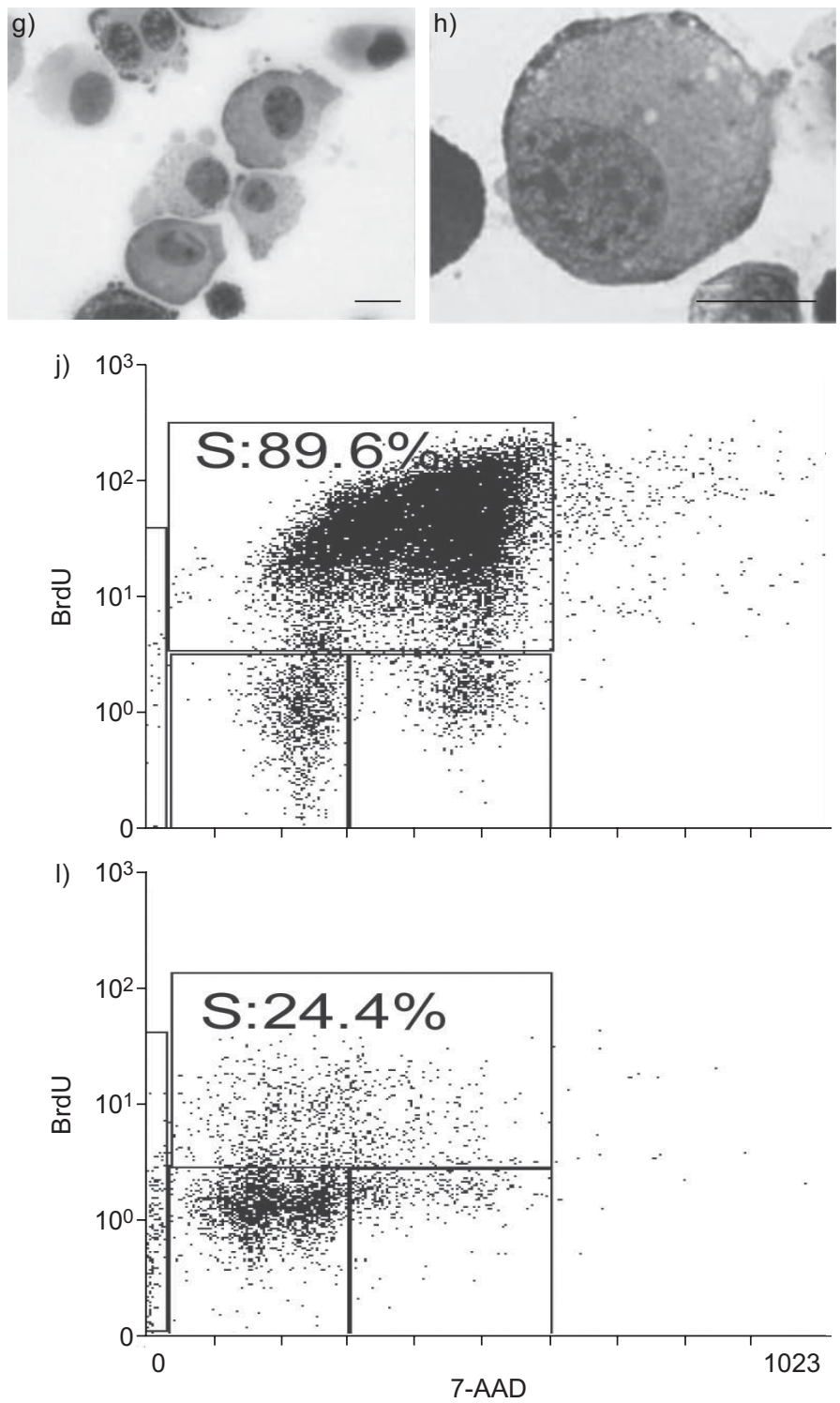

FIGURE 2. Morphology and fluorescence-activated cell sorting analysis of murine bone marrow mesenchymal stem cells (BMMSCs). a-h) Photomicrograph of bone marrow-adherent cells stained with Diff-Quik. a and b) 2-h adherent CD11b+/CD31+/CD45+ cells. c and d) 9-day adherent CD11b+/ CD45+ cells. e and f) 2-h adherent CD11b-CD45-CD31- cells (2-h adherent BMMSCs). $g$ and h) 9-day adherent CD11b-CD45- cells (9-day cultured BMMSCs). Scale bars=200 $\mu \mathrm{m}$. i-l) Cell cycle analysis. Incorporated bromodeoxyuridine (BrdU) (with fluorescein isothiocyanate anti-BrdU) and total DNA content (with 7-aminoactinomycin (7-AAD)) were measured by flow cytometry. Gates applied to the BrdU versus 7-AAD plot indicate cell subsets that were apoptotic or resided in G0/G1, S or G2/M phase in the cell cycle, respectively. i) 2-h adherent whole bone marrow cells. Apoptotic cells: 0.2\%; G0/G1: 57.8\%; S: 35.5\%; G2/M: 4.7\%. j) 2-h adherent BMMSCs (2-h adherent CD11b-CD45-CD31- cells). k) 9-day adherent whole bone marrow cells. Apoptotic cells: 0.3\%; G0/G1: 66.9\%; S: 23.6\%; G2/M: 9.3\%. Apoptotic cells: 0.1\%; G0/G1: 5.1\%; S: 89.6\%; G2/M: 3.1\%. I) 9-day cultured BMMSCs (9-day adherent CD11b-CD45- cells). Apoptotic cells: 4.2\%; G0/G1: 60.1\%; S: 24.4\%; G2/M: 9.6\%. The 7-AAD signal was acquired in linear mode. Data shown are from one representative of two independent experiments. 
fraction of 9-day cultured BMMSCs in the S phase $(24.4 \%)$ was markedly reduced compared with 2-h adherent BMMSCs (89.6\%; fig. $2 \mathrm{j}$ and 1 ). According to the results of the bivariate cell cycle analysis, therefore, the proliferative capacity of BMMSCs decreases during cultivation.

Previous studies have reported that BMMSCs express several stem cell markers, representing their "stemness". In order to determine whether the expression levels of stem cell markers are altered in the process of BMMSC culture, we quantified mRNA levels for Oct3/4, Nanog, Sox2, SSEA-1 and Rex-1, all of which were reported to be associated with BMMSCs [14, 15]. Gene expressions of these stem cell markers in BMMSCs are uniformly downregulated during cell culture by a 1.9- to 137.9-fold decrease on average (fig. 3a-e). Furthermore, such downregulation of stem cell gene expressions were maintained in 8 month-cultured BMMSCs (fig. 2a in the online supplementary material).

Expression of a broad spectrum of chemokine receptors that play an important role in cellular recruitment to sites of injury have been reported for BMMSCs [16-19]. The expressions of all chemokine receptors examined, including CXCR1-6, CCR1-9 and CX3CR1, were downregulated as a function of culture duration (fig. 3f).

To evaluate the possible influence of 2-h adherent BMMSC or 9-day cultured BMMSC infusions on lung fibrosis, we examined the expression of type I procollagen, $\alpha$-smooth muscle actin ( $\alpha$-SMA) and transforming growth factor (TGF)- $\beta$. 9-day cultured BMMSCs expressed type I procollagen at a level 1.92-fold that of 2-h adherent BMMSCs (fig. 3a in the online supplementary material). Moreover, long-term cultured (8 months) BMMSCs showed an almost 18-fold increase compared with 2-h adherent BMMSCs (data not shown) and the expression of $\alpha$-SMA in 9-day cultured BMMSCs was higher than that in 2-h adherent BMMSCs (fig. 3b in the online supplementary material), indicating the possibility that BMMSCs gradually acquire fibroblast-like characteristics during cultivation. Next, the expression of TGF- $\beta$, a cytokine known to promote lung fibrosis, was examined. After adjusting for the amount of total RNA in each donor cell, 9-day cultured BMMSCs expressed 1.46 times as much TGF- $\beta$ as 2-h adherent BMMSCs (fig. $3 c$ in the online supplementary material). The expressions of TGF- $\beta$ in 9-day cultured BMMSCs were always higher than those of 2-h adherent BMMSCs in three independent experiments.

Because acquisition of tumorigenicity is one of the possible obstacles to successful and safe BMMSC treatment, we also evaluated whether the expression level of oncogenic marker cMyc was altered during cell cultivation. Even within 10 days of culture, gene expression of c-Myc was upregulated (fig. 3d in the online supplementary material), and this was further enhanced in long-term cultured BMMSCs (fig. 2b in the online supplementary material). These results indicate that 9-day cultured BMMSCs might acquire tumourigenic property during the process of cellular alteration in 2-h adherent BMMSCs.

\section{In vivo effects of administration of 2-h adherent BMMSCs or 9-day cultured BMMSCs on bleomycin-induced fibrotic lung injury}

To evaluate the in vivo effects of 2-h adherent BMMSCs and 9day cultured BMMSCs on bleomycin-induced lung injury, these cells were administered 3 days after bleomycin challenge. In more than one-half of mice given 2-h adherent BMMSCs or 9-day cultured BMMSCs, weight was restored and the animals survived, whereas only three out of 10 recovered from weight loss and were still alive at day 14 in control mice (fig. $4 \mathrm{a}-\mathrm{c}$ ). Survival curves indicated that mice treated with 2-h adherent BMMSCs or 9-day cultured BMMSCs exhibited prolonged survival compared with control mice (control 52\% versus 2-h adherent BMMSCs $80 \%(\mathrm{p}<0.05)$, and versus 9-day cultured BMMSCs 74\%) (fig. 4d). Thus, there was a statistically significant difference between the control group and the 2-h adherent BMMSC but not the 9-day cultured BMMSC group.

BAL fluid collected from each group showed severe alveolar haemorrhage in five out of six control mice, but only in one out of five mice infused with 2-h adherent BMMSCs or 9-day cultured BMMSCs, respectively (fig. 5a). Furthermore, administration of 2-h adherent BMMSCs or 9-day cultured BMMSCs attenuated the increase in both total BAL cell counts and neutrophil counts 10 days after bleomycin injection (fig. $5 b$ and c).

In order to assess the antifibrotic role of 2-h adherent BMMSCs and 9-day cultured BMMSCs in bleomycin-induced pulmonary fibrosis, we examined type I procollagen mRNA expression in the lungs. Our data revealed that transcription of type I procollagen was downregulated in mice receiving 2-h adherent BMMSCs or 9-day cultured BMMSCs compared with mice treated with PBS alone (fig. 5d). In addition, lung collagen quantification demonstrated nearly equal decreases in collagen deposition in the lungs treated with 2-h adherent BMMSCs or 9-day cultured BMMSCs compared with those with PBS alone (fig. 5e). Lung histopathology on haemotoxylin and eosin staining (fig. 5f-h), the fluorescence intensity of type I collagen on immunofluorescent staining (fig. 4 in the online supplementary material) and the Ashcroft score, a numerical fibrotic scale (fig. 5 in the online supplementary material) [20], confirmed that administration of BMMSCs attenuated bleomycin-induced pulmonary fibrosis. The sections shown in figure $5 \mathrm{f}-\mathrm{g}$ and the online supplementary figure 4 were prepared from the most damaged lung, which was ranked by several factors, including total BAL cell counts, the number of neutrophils in BAL, the expression level of type I procollagen and tumour necrosis factor- $\alpha$ in the lung.

These data suggest that infusion of 2-h adherent BMMSCs or 9day cultured BMMSCs after bleomycin administration results in reduction of inflammation and fibrosis in the lung.

\section{DISCUSSION}

Mesenchymal stem cells are currently promising candidates for cell-based therapy. Human mesenchymal stem cells have already been used in clinical trials for repairing various types of organ injuries [21], including metabolic [22] or haematological diseases [23]. Further applications are being developed using animal models, and may soon become available at the bedside. As for the lung diseases, ALI and ARDS are still associated with high mortality, presently lacking effective treatment strategies, and BMMSC-based treatment has been investigated to be an alternative to conventional remedies.

Almost all studies investigating the restorative capacity of BMMSCs have used culture-expanded cells, most likely because of their established regenerative properties in vitro and ease of cell harvesting. Conversely, some disadvantages of 

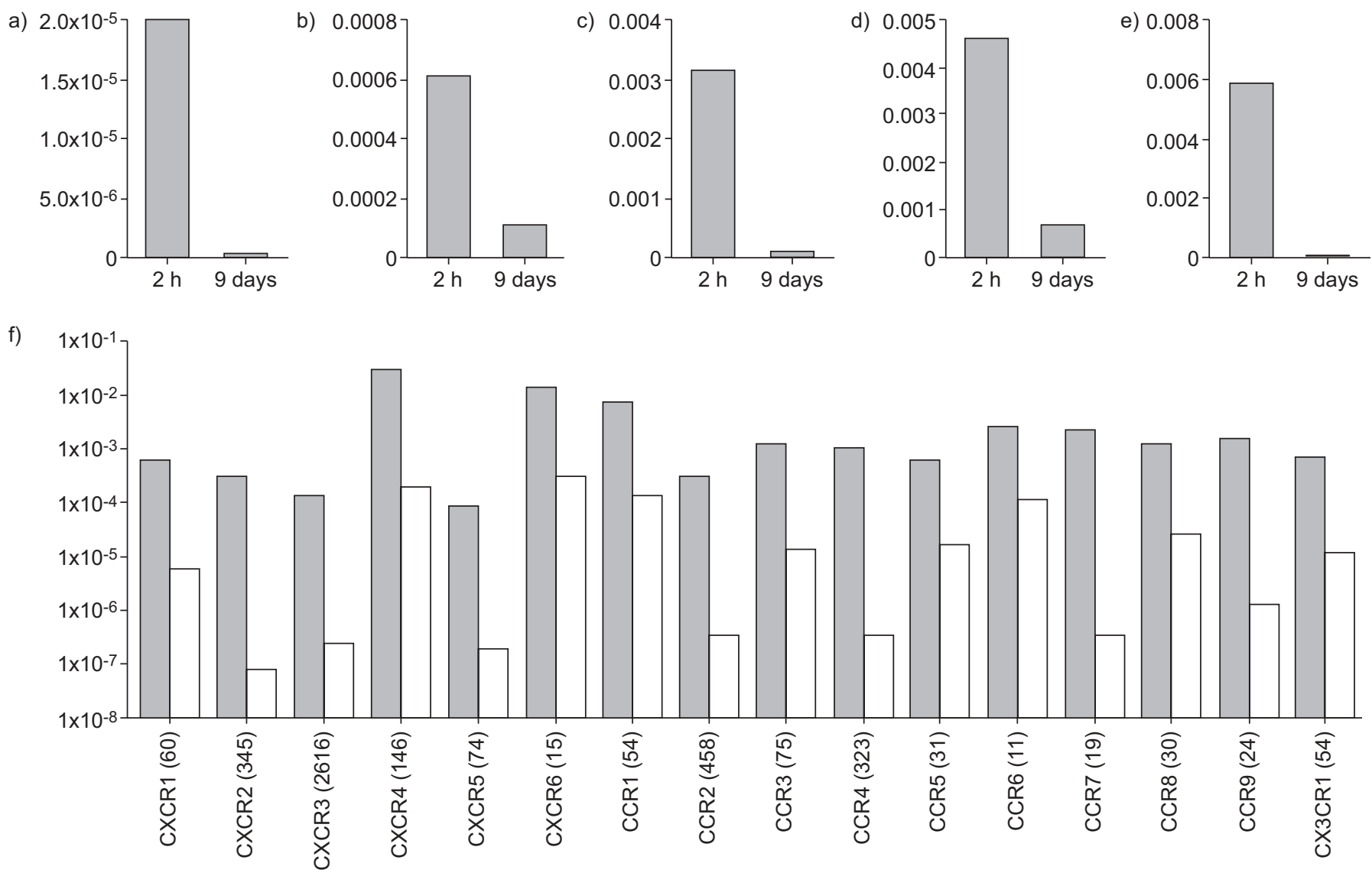

FIGURE 3. Real-time PCR of stem cell marker and chemokine receptor expression by 2-h adherent bone marrow mesenchymal stem cells (BMMSCs) and 9-day cultured BMMSCs. 9-day cultured BMMSCs showed lower expression of stem cell markers a) Oct3/4 (137.9-fold difference), b) Nanog (1.9-fold difference), c) Sox2 (37.4-fold difference), d) SSEA-1 (17.6-fold difference) and e) Rex-1 (137.9-fold difference), compared with 2-h adherent BMMSCs. f) Chemokine receptors CXCR1-6, CCR1-9 and CX3CR1 were expressed in both 2-h adherent BMMSCs $(\square)$ and 9-day cultured BMMSCs $(\square)$ at the mRNA level, although expression was very low. 9-day cultured BMMSCs showed lower expression of all chemokine receptors compared with 2-h adherent BMMSCs. The expression level is normalised to the level of glyceraldehyde 3-phosphate dehydrogenase. We repeated these experiments three times and similar results were obtained. Data shown are from one representative of three independent experiments. The average ratios of the expressions of chemokine receptors in 2-h adherent BMMSCs to 9-day cultured BMMSCs are shown in parentheses.

the BMMSC-culture process have been reported with respect to culture conditions detrimental for cell transplantation and resulting in biological alterations of the cells. In cases where fetal calf serum has been used, there are risks of contamination and immunological reactions against xenogeneic serum antigens [5], which can be eliminated by using autologous human serum. However, because the amount of available blood is limited, it seems logistically problematic to expand human mesenchymal stem cells with autologous human serum.

Another issue for BMMSC culture is the unexpected modifications in the biology of cultured cells. It has been demonstrated that BMMSCs begin changes culminating in senescence from the start of culturing, with decreases in their proliferative capacity, differentiation, homing ability [2,4] and telomere length [24], as well as acquisition of potentially tumorigenic phenotypes with chromosomal abnormalities, which can result in sarcoma formation in vivo $[6,7]$. According to these data, it seems that culture duration should be shortened as much as is possible for cell-based therapy. If 2-h adherent BMMSCs are equally as effective in terms of grafting as 9-day cultured BMMSCs, serum requirements should be sufficiently reduced such that the limited amounts of autologous serum available could be adequate.

In the present study, we found that multipotent mesenchymal stromal cells were contained in short-term cultured adherent bone marrow cells, and the inducibly differentiated cells showed morphological similarities with 9-day cultured BMMSCs, but not with the cells of myeloid/endothelial lineages. It is generally accepted that BMMSCs are plastic-adherent cells which are negatively selected by haematopoietic, myeloid lineage and endothelial markers [13], as evidenced by their ability to differentiate into mesenchymal lineages. In fact, the separately cultivated 2-h adherent CD45-CD11b-CD31- bone marrow cells uniformly developed into extended fibroblast-like cells resembling 9-day cultured BMMSCs. Based on these findings, we defined the short-term cultured adherent CD45-CD11b-CD31bone marrow cells as 2-h adherent BMMSCs.

A study of the biological differences between 2-h adherent BMMSCs and 9-day cultured BMMSCs, i.e. those examined in this study, documented the acquisition of several culture timedependent cellular characteristics not desirable for cell 

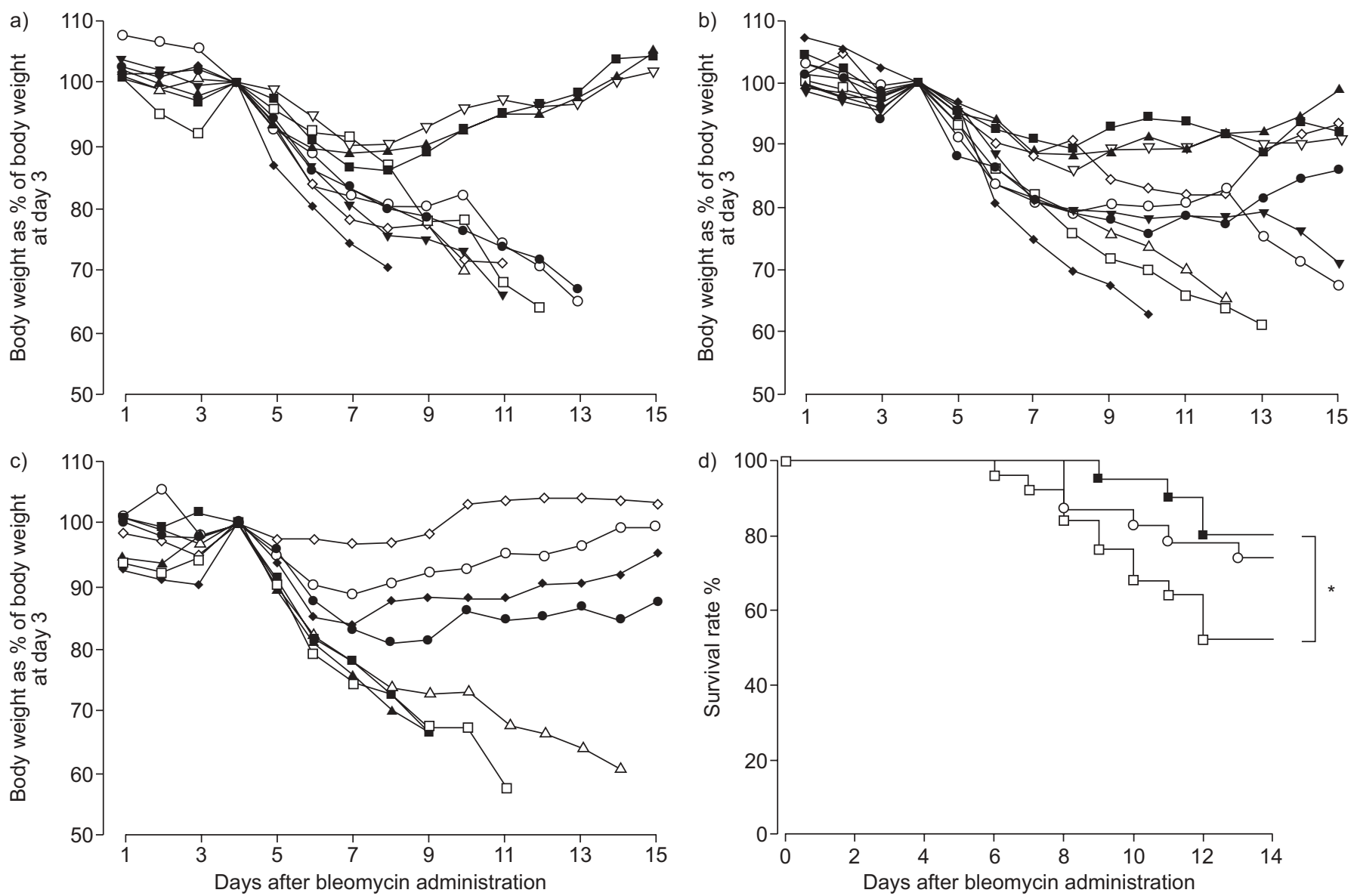

FIGURE 4. Effect of 2-h adherent bone marrow mesenchymal stem cells (BMMSCs) and 9-day cultured BMMSCs on body weight reduction and mortality in mice exposed to bleomycin. a-c) Time course of body weight changes of mice exposed to bleomycin followed by administration of b) 2-h adherent BMMSCs (five (50\%) out of 10 mice recovered), c) 9-day cultured BMMSCs (four (50\%) out of eight mice recovered) or a) PBS alone (three (30\%) out of 10 mice recovered). The relative body weight is expressed as the percentage of relative body weight at day 3 (the day of transfer). Data shown are from one representative of three independent experiments. d) Results are presented as a Kaplan-Meier survival curve for mice receiving $5 \times 10^{5}$ 2-h adherent BMMSCs $(\boldsymbol{\square} ; n=20), 5 \times 10^{5}$ 9-day cultured BMMSCs $(O$; $n=23)$, or PBS alone ( $\square$; $n=25)$. Survival data shown are from three independent experiments, which showed similar survival curves. $*$ : $p<0.05$.

transplantation. First, enlarged cell size was likely to increase the risk of embolism; indeed, two out of 10 mice died immediately after BMMSC administration and we supposed the cause of their death was pulmonary embolism. Previous studies have also recognised this risk [25].

For successful cell grafting, cells need to maintain not only viability, but also their stemness and the ability to traffic into appropriate sites from the circulation [16-19]. Our data showed that 2-h adherent BMMSCs had higher growth capacity and enhanced expression levels of stem cell markers and chemokine receptors than 9-day cultured BMMSCs, indicating that they possessed putative advantages for cellgrafting over 9-day cultured BMMSCs.

In addition, the enhanced expressions of type I procollagen and TGF- $\beta$ in 9-day cultured BMMSCs suggested that these cells may cause adverse effects on lung fibrosis. Furthermore, upregulation of c-Myc expression during cultivation reminded us of the risk of tumour formation in the use of expanded BMMSC.

Favourable cellular properties of 2-h adherent BMMSCs for cell grafting prompted us to examine whether they could replace 9-day cultured BMMSCs for the management of fibrotic lung injury. In general, four stages characterise the clinical course of ARDS: injury ( $\sim 6 \mathrm{~h})$ followed by apparent stability $(12-48 \mathrm{~h})$, but then respiratory insufficiency and, finally, the terminal stage. Diagnosis of ARDS is often made only at the stage of respiratory insufficiency [26]. Therefore, the suitability of BMMSC transplantation for fatal lung injury requires further verification using a stricter protocol, avoiding early administration of the cells. To make our experimental model more relevant to the clinical setting, we injected donor cells 3 days after bleomycin challenge without myelosuppression, because inflammation and fibrosis were already manifested in the lung at this stage. The results showed that 2-h adherent BMMSCs mediated protective effects for alveolar haemorrhage, inflammatory cell influx and progression of fibrogenesis, and prevented weight loss and mortality equally well or better than 9-day cultured BMMSCs.

Although the principal mechanisms by which BMMSCs contribute to lung repair remain undetermined, there are many studies indicating the homing ability of bone marrow cells to injured lung and active differentiation into many types of cells $[8,9,27,28]$. In contrast, two recent studies failed to observe 
a)

PBS

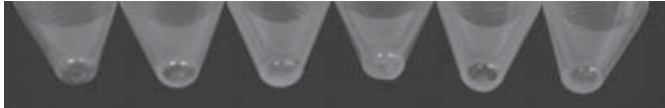

2-h BMMSCs

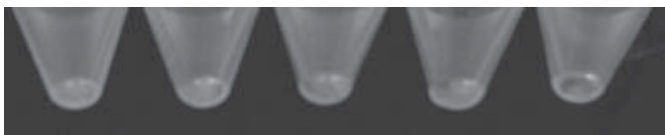

9-day BMMSCs
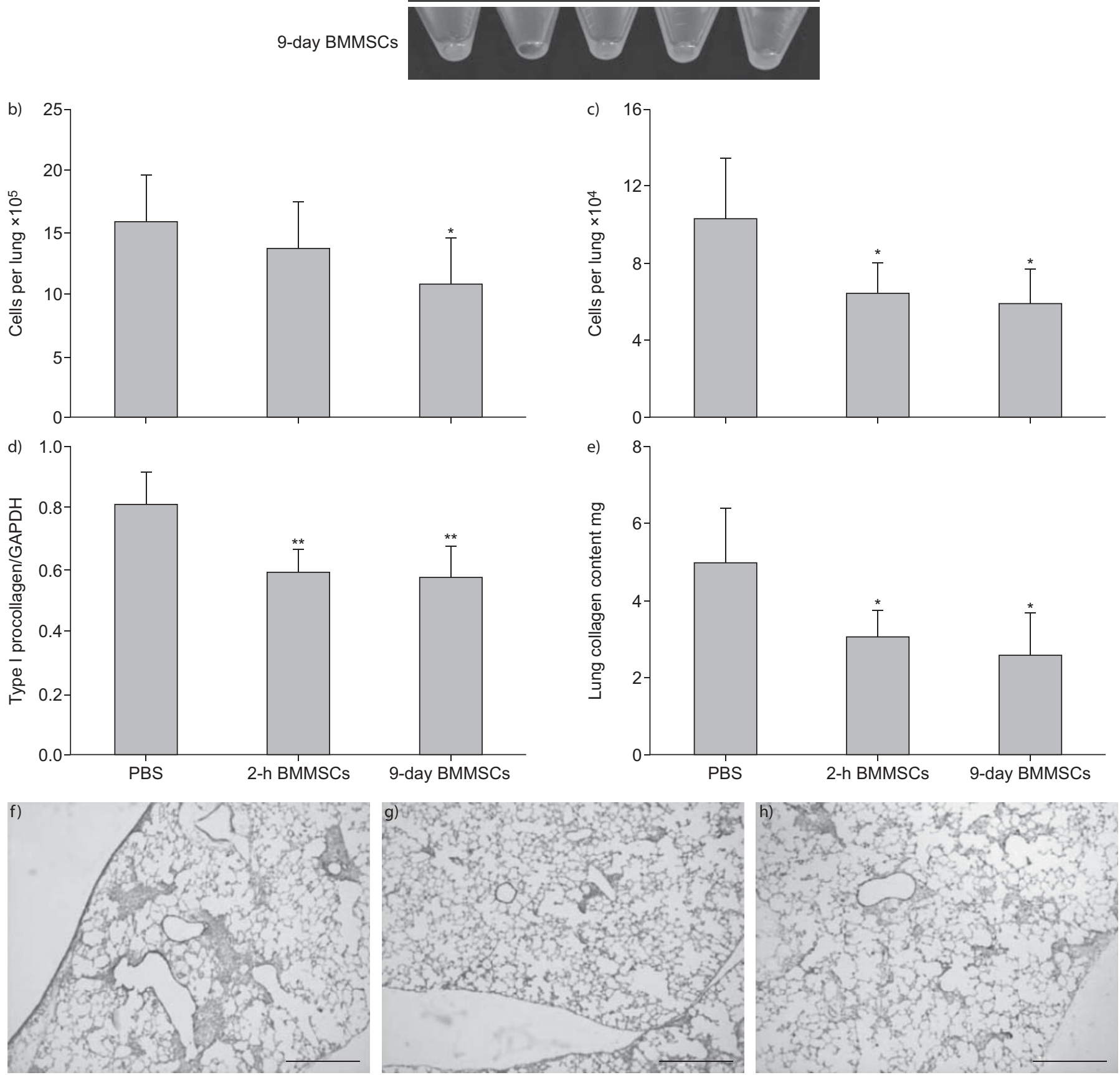

FIGURE 5. Protective effect of 2-h adherent bone marrow mesenchymal stem cells (BMMSCs) and 9-day cultured BMMSCs in bleomycin-induced pulmonary inflammation and fibrosis. a) Photographs of cells collected from each group via bronchoalveolar lavage (BAL); $n=6$ in PBS control group, and $n=5$ in 2-h adherent BMMSC and 9-day cultured BMMSC groups. b) Total BAL cell counts. c) Gr-1 positive cell (neutrophil) counts analysed by flow cytometry. d) Type I procollagen mRNA expression in the lungs 10 days after injection of bleomycin followed by 2-h adherent BMMSC transfer $(n=5)$ or 9-day cultured BMMSC transfer $(n=5)$ compared with PBS control $(n=6)$ as determined by real-time PCR. b-d) Data shown are from one representative of three independent experiments. e) Lung collagen content at day 10 after injection of bleomycin followed by 2-h adherent BMMSC transfer $(n=5)$ or 9 -day cultured BMMSC transfer $(n=5)$ compared with PBS control $(n=5)$. Data are expressed as mean \pm SD. *: $p<0.05$; and **: $p<0.01$ versus PBS control group. $f-g)$ Histological findings by haemotoxylin and eosin staining. Each section was obtained from lung tissue 10 days after intratracheal bleomycin administration followed by f) PBS infusion, g) 2-h adherent BMMSC transfer or h) 9-day cultured BMMSC transfer. Scale bars $=500 \mu \mathrm{m}$. 
bone marrow transplantation-induced lung reconstitution in transgenic mice infused with surfactant protein $\mathrm{C}$-enhanced green fluorescent protein transgenic bone marrow cells $[29,30]$.

However, accumulating evidence suggests that BMMSCinduced tissue protection is provided not by donor cell replacement of damaged lung cells, but rather by humoral factors released from the injected cells, such as growth factors and anti-inflammatory cytokines [11, 31]. Further research must be required to elucidate the mechanisms underlying the effects of BMMSC transplantation on fibrotic lung injury, and will provide more efficient therapeutic strategies.

In summary, our data demonstrated that, during cultivation, BMMSCs acquire properties unfavourable for cell-based therapy; moreover, the ability of 2-h adherent BMMSCs to ameliorate lung damage was equal to or better than 9-day cultured BMMSCs in vivo.

We believe that our findings are valuable for further investigation to resolve the ethical and biological issues surrounding the clinical use of BMMSCs, not only for lung diseases but also for various other organ disorders.

\section{STATEMENT OF INTEREST}

None declared.

\section{REFERENCES}

1 Friedenstein AJ, Gorskaja JF, Kulagina NN. Fibroblast precursors in normal and irradiated mouse hematopoietic organs. Exp Hematol 1976; 4: 267-274.

2 Bonab MM, Alimoghaddam K, Talebian F, et al. Aging of mesenchymal stem cell in vitro. BMC Cell Biol 2006; 7: 14.

3 Chachques JC, Herreros J, Trainini J, et al. Autologous human serum for cell culture avoids the implantation of cardioverterdefibrillators in cellular cardiomyoplasty. Int J Cardiol 2004; 95: Suppl. 1, S29-S33.

4 Rombouts WJ, Ploemacher RE. Primary murine MSC show highly efficient homing to the bone marrow but lose homing ability following culture. Leukemia 2003; 17: 160-170.

5 Tuschong L, Soenen SL, Blaese RM, et al. Immune response to fetal calf serum by two adenosine deaminase-deficient patients after $\mathrm{T}$ cell gene therapy. Hum Gene Ther 2002; 13: 1605-1610.

6 Miura M, Miura Y, Padilla-Nash HM, et al. Accumulated chromosomal instability in murine bone marrow mesenchymal stem cells leads to malignant transformation. Stem Cells 2006; 24: 1095-1103.

7 Tolar J, Nauta AJ, Osborn MJ, et al. Sarcoma derived from cultured mesenchymal stem cells. Stem Cells 2007; 25: 371-379.

8 Ortiz LA, Gambelli F, McBride C, et al. Mesenchymal stem cell engraftment in lung is enhanced in response to bleomycin exposure and ameliorates its fibrotic effects. Proc Natl Acad Sci USA 2003; 100: 8407-8411.

9 Rojas M, Xu J, Woods CR, et al. Bone marrow-derived mesenchymal stem cells in repair of the injured lung. Am J Respir Cell Mol Biol 2005; 33: 145-152.

10 Ishizawa K, Kubo H, Yamada M, et al. Bone marrow-derived cells contribute to lung regeneration after elastase-induced pulmonary emphysema. FEBS Lett 2004; 556: 249-252.

$11 \mathrm{Xu} \mathrm{J}$, Woods CR, Mora AL, et al. Prevention of endotoxin-induced systemic response by bone marrow-derived mesenchymal stem cells in mice. Am J Physiol Lung Cell Mol Physiol 2007; 293: L131-L141.
12 Phinney DG, Kopen G, Isaacson RL, et al. Plastic adherent stromal cells from the bone marrow of commonly used strains of inbred mice: variations in yield, growth, and differentiation. J Cell Biochem 1999; 72: 570-585.

13 Kotton DN, Fine A. Derivation of lung epithelium from bone marrow cells. Cytotherapy 2003; 5: 169-173.

14 Greco SJ, Liu K, Rameshwar P. Functional similarities among genes regulated by OCT4 in human mesenchymal and embryonic stem cells. Stem Cells 2007; 25: 3143-3154.

15 Guillot PV, Gotherstrom C, Chan J, et al. Human first-trimester fetal MSC express pluripotency markers and grow faster and have longer telomeres than adult MSC. Stem Cells 2007; 25: 646-654.

16 Honczarenko M, Le Y, Swierkowski M, et al. Human bone marrow stromal cells express a distinct set of biologically functional chemokine receptors. Stem Cells 2006; 24: 1030-1041.

17 Sordi V, Malosio ML, Marchesi F, et al. Bone marrow mesenchymal stem cells express a restricted set of functionally active chemokine receptors capable of promoting migration to pancreatic islets. Blood 2005; 106: 419-427.

18 Schenk S, Mal N, Finan A, et al. Monocyte chemotactic protein-3 is a myocardial mesenchymal stem cell homing factor. Stem Cells 2007; 25: 245-251.

$19 \mathrm{Ji} \mathrm{JF}, \mathrm{He} \mathrm{BP}$, Dheen ST, et al. Interactions of chemokines and chemokine receptors mediate the migration of mesenchymal stem cells to the impaired site in the brain after hypoglossal nerve injury. Stem Cells 2004; 22: 415-427.

20 Ashcroft T, Simpson JM, Timbrell V. Simple method of estimating severity of pulmonary fibrosis on a numerical scale. J Clin Pathol 1988; 41: 467-470.

21 Kawada H, Fujita J, Kinjo K, et al. Nonhematopoietic mesenchymal stem cells can be mobilized and differentiate into cardiomyocytes after myocardial infarction. Blood 2004; 104: 3581-3587.

22 Koc ON, Day J, Nieder M, et al. Allogeneic mesenchymal stem cell infusion for treatment of metachromatic leukodystrophy (MLD) and Hurler syndrome (MPS-IH). Bone Marrow Transplant 2002; 30: 215-222.

23 Koc ON, Gerson SL, Cooper BW, et al. Rapid hematopoietic recovery after coinfusion of autologous-blood stem cells and culture-expanded marrow mesenchymal stem cells in advanced breast cancer patients receiving high-dose chemotherapy. J Clin Oncol 2000; 18: 307-316.

24 Banfi A, Bianchi G, Notaro R, et al. Replicative aging and gene expression in long-term cultures of human bone marrow stromal cells. Tissue Eng 2002; 8: 901-910.

25 Schrepfer S, Deuse T, Reichenspurner H, et al. Stem cell transplantation: the lung barrier. Transplant Proc 2007; 39: 573-576.

26 Balk R, Bone RC. The adult respiratory distress syndrome. Med Clin North Am 1983; 67: 685-700.

27 Abe S, Lauby G, Boyer C, et al. Transplanted BM and BM side population cells contribute progeny to the lung and liver in irradiated mice. Cytotherapy 2003; 5: 523-533.

28 Kotton DN, Ma BY, Cardoso WV, et al. Bone marrow-derived cells as progenitors of lung alveolar epithelium. Development 2001; 128: 5181-5188.

29 Chang JC, Summer R, Sun X, et al. Evidence that bone marrow cells do not contribute to the alveolar epithelium. Am J Respir Cell Mol Biol 2005; 33: 335-342.

30 Kotton DN, Fabian AJ, Mulligan RC. Failure of bone marrow to reconstitute lung epithelium. Am J Respir Cell Mol Biol 2005; 33: 328-334.

31 Togel F, Hu Z, Weiss K, et al. Administered mesenchymal stem cells protect against ischemic acute renal failure through differentiation-independent mechanisms. Am J Physiol Renal Physiol 2005; 289: F31-F42. 